\title{
Análise de Cointegração com Threshold nos Mercados Exportadores de Mel Natural no Brasil
}

\author{
Manoel Pedro da Costa Júnior ${ }^{1}$, Ahmad Saeed Khan², \\ Eliane Pinheiro de Sousa ${ }^{3}$ e Patrícia Verônica Pinheiro Sales Lima ${ }^{4}$
}

Resumo: Este trabalho analisa a integração espacial dos mercados exportadores de mel natural no Brasil, levando-se em consideração a presença de custos de transação, uma vez que tais custos podem gerar assimetrias nos relacionamentos de preços entre os mercados analisados. Para atender a este objetivo, utilizou-se o método analítico de cointegração com threshold por meio da estimação dos modelos TAR e M-TAR. Os dados foram obtidos no Ministério de Desenvolvimento, Indústria e Comércio Exterior no período de janeiro de 2002 a julho de 2011. Os resultados indicam a presença de assimetrias na transmissão do preço estabelecido no mercado central, representada pelo Rio Grande do Sul, e os demais estados considerados. Ademais, choques negativos e de baixa magnitude foram excluídos, diferente dos choques positivos. Portanto, conclui-se que os custos de transação influenciam a interação espacial entre os mercados.

Palavras-chaves: Integração de mercados; Cointegração com threshold; Mel natural.

Abstract: This study analyzes the spatial integration of exporting markets of raw honey in Brazil, taking into account the presence of transaction costs, since such costs may generate asymmetries in the relationships of prices among the markets under analysis. In order to achieve this goal, the analytical method of threshold cointegration by means of the estimation of the TAR and M-TAR models was used. The data were obtained in the Ministry of Development, Industry and Foreign Commerce for the period from January 2002 to July 2011. The results indicate the presence of asymmetries in the transmission of the price

1. Professor do Instituto Federal de Educação, Ciência e Tecnologia do Sertão Pernambucano. Petrolina, PE, Brasil. E-mail: pedrojunior_noronha@yahoo.com.br

2. Professor Titular do Departamento de Economia Agrícola da Universidade Federal do Ceará. Fortaleza, CE, Brasil. E-mail: saeed@ufc.br

3. Professora Adjunta do Departamento de Economia da Universidade Regional do Cariri. Crato, CE, Brasil. E-mail: pinheiroeliane@hotmail.com

4. Professora Adjunta do Departamento de Economia Agrícola da Universidade Federal do Ceará. Fortaleza, CE, Brasil. E-mail: pvpslima@gmail.com 
established in the central market, represented by Rio Grande do Sul, and the other states under consideration. In addition, negative and low magnitude shocks were excluded differently from the positive shocks. Therefore, one may conclude that the transaction costs influence the spatial interaction among the markets.

Key-words: Market integration; Threshold cointegration; Raw honey.

http://dx.doi.org/10.1590/1234-56781806-9479005302007

\section{Introdução}

O mel brasileiro conquistou maiores níveis de aceitação no mercado internacional em virtude da adequação a processos capazes de minimizar efeitos que provoquem redução na qualidade do produto, com destaque para a presença de defensivos agrícolas. Com relação ao cumprimento das especificidades da demanda dos consumidores que exigem alimentos sem vestígios de resíduos redutores de qualidade, o Brasil, se comparado à China (uma das nações que mais produzem e comercializam mel), possui vantagens que podem ser atribuídas principalmente à mata nativa (MATOS, 2005).

Segundo dados da FAO (2011), nas últimas décadas do século XX e na primeira deste século, o Brasil esteve entre os 11 países que mais exportaram mel natural. Os estados brasileiros exportadores de mel natural são São Paulo, Ceará, Rio Grande do Sul, Santa Catarina e Piauí. As regiões Sudeste e Sul são as maiores exportadoras, com participações de $36,12 \%$ e $32,56 \%$, respectivamente, da quantidade exportada de mel no Brasil, em 2010, conforme dados do Ministério do Desenvolvimento, Indústria e Comércio Exterior (BRASIL, 2011), seguida pela região Nordeste, responsável por $31,26 \%$ da quantidade exportada no País. Após o grande exportador, que é o estado de São Paulo, Piauí e Rio Grande do Sul se apresentam como os territórios brasileiros que mais exportaram mel natural em 2010. De acordo com essa base de dados, as regióes Norte e CentroOeste brasileiras apresentam inexpressiva participação no total de mel natural exportado pelo Brasil, em que contribuem com cerca de $0,01 \%$ do total comercializado pelo mercado brasileiro.

De posse desses dados, verifica-se que o mel natural desempenha importante papel na economia brasileira, visto que sua produção e exportação estão distribuídas espacialmente em todo território brasileiro, contribuindo para a geração de emprego e renda. Em conformidade com Khan et al. (2014), a apicultura é uma atividade praticada predominantemente por produtores, tendo se configurado numa alternativa para diversificação da fonte de renda nas pequenas propriedades rurais. De cada família que trabalha na apicultura, em média, 2,1 pessoas se envolvem na atividade.

Neste sentido, julga-se importante compreender como acontece o processo de integração desse produto nos principais estados exportadores. De acordo com Meyer (2004), a integração de mercado pode ser definida como o grau de transmissão de preços entre mercados espacialmente separados. As commodities são alvos frequentes dos estudos de integração de mercados que, no decorrer da investigação, mensura se os choques de oferta e demanda em uma dada região podem ser transmitidos para outra.

As análises dos relacionamentos de preços entre os mercados, durante muitos anos, foram 
baseadas exclusivamente na Lei do Preço Único (LPU). De acordo com Krugman e Obstfeld (2005), essa lei postula que, sob livre concorrência e na ausência de custos de transporte e barreiras ao comércio, bens idênticos devem ser vendidos ao mesmo preço em diferentes localidades, quando seus preços são cotados em termos da mesma moeda. No entanto, esta teoria não incorpora nenhuma forma de presença de custos de transação, ou seja, não contempla custos de transporte e de transferência. Portanto, o rompimento dos pressupostos da LPU permite destacar que pode haver custos relacionados com a distância física entre os mercados. Por exemplo, os estados de São Paulo e Ceará estão separados geograficamente entre si e com relação aos demais estados exportadores, o que pode incorrer em custos de transporte diferenciados.

Para Mattos (2008), os custos de transação não desprezíveis talvez possam contribuir com a redução da transmissão de choques de preços entre mercados espacialmente separados. Como consequência, tem-se a redução da possibilidade de os mercados se tornarem economicamente integrados, uma vez que a transmissão de preços somente ocorre quando os ganhos com a arbitragem superam os custos de transação.

Estudos tradicionais que avaliam o processo de transmissão de preços têm sido realizados para várias culturas agropecuárias no Brasil, como por exemplo, algodão, soja, trigo, cacau, leite, carnes, frutas (melão, maracujá), castanha de caju etc. Entretanto, não se encontrou, na literatura econômica nacional nem internacional, essa aplicação para os mercados de mel natural, apesar da expressiva relevância desempenhada por esse produto para a economia brasileira.

Assim, além de se tratar de um estudo pioneiro, este artigo incorpora os custos de transação, uma vez que a presença de tais custos pode ocasionar assimetrias ou mesmo impedir o processo de transmissão de preços, que não são levados em consideração nos métodos convencionais de séries temporais. Conforme Barrett (2001), se houver custos de transação no mercado em análise, porém esses não forem devida- mente computados, as avaliações realizadas não traduzem o verdadeiro relacionamento espacial entre tais mercados, já que esses custos passam a fazer parte do termo de erro das regressões. Portanto, os parâmetros estimados serão viesados e inconsistentes.

A análise sobre a transmissão de preços considerando a existência de custos de transação no mercado brasileiro de mel assume papel fundamental, tendo em vista que o mercado de mel apresenta uma especificidade relacionada ao nível de sensibilidade desse produto em assimilar características locais, isto é, desde o processo de manejo no apiário, passando pela colheita, armazenagem e comercialização, podendo gerar reduções na qualidade do mel. Acredita-se que a proximidade geográfica do apicultor com os grandes entrepostos responsáveis pela industrialização e comercialização do mel produzido possa contribuir para minimizar as perdas de produtividade e manter a qualidade desse produto. Outra peculiaridade do mercado de mel diz respeito à susceptibilidade em absorver características do meio ambiente representadas no cheiro, na cor e na viscosidade.

Além dessas especificidades existentes no mercado de mel, também se deve destacar que o conhecimento sobre a formação dos preços e seu processo de transmissão entre mercados reveste-se de importância para o setor produtor e exportador de mel natural no Brasil, podendo contribuir para um melhor desempenho de sua cadeia produtiva.

A partir dos resultados que serão obtidos por esta pesquisa, haverá a possibilidade de verificar se a análise de integração entre mercados do mel natural é eficiente do ponto de vista dos preços desse produto. De acordo com Mattos (2008, p. 31), "os autores que defendem que a integração seja vista como um conceito pautado na "comerciabilidade" dos produtos, e não na interdependência dos preços, mostram-se descrentes quanto à real contribuição de estudos que buscam verificar se mercados são ou não integrados".

Portanto, a análise das variações de preços de exportação do mercado brasileiro de mel 
natural permitirá elencar uma série de informações que possibilitarão compreender com mais clareza sobre como tais preços são constituídos. Salienta-se, ainda, que a implementação deste estudo fornecerá subsídios que ajudarão no processo de formulação de políticas públicas, com o intuito de conquistar maior competitividade do mel natural produzido no Brasil. Em outros termos, a identificação do estado que atua como mercado central formador de preços facilitará o fluxo de informações, buscando minimizar os gastos públicos e gerar uma alocação eficiente dos recursos, contribuindo, dessa forma, para a melhoria de medidas de políticas públicas destinadas ao setor apícola.

Em face dessas considerações, este trabalho se propõe a analisar a integração espacial dos mercados exportadores de mel natural no Brasil, levando em consideração a presença de custos de transação. Especificamente, pretende-se identificar o mercado central formador de preços de mel natural e verificar se existem diferenças no processo de transmissão de preços entre regióes localizadas espacialmente mais distantes entre si.

Além destes relatos introdutórios, o artigo contém seis seções. A segunda seção realiza a descrição do mercado de mel natural no Brasil. Os fundamentos teóricos sobre integração espacial e custos de transação estão apresentados na terceira seção. A revisão de literatura contendo estudos que incorporaram os efeitos dos custos de transação sobre o processo de ajustamento de preços está exposta na quarta seção. Em seguida, são mostrados os métodos analíticos e a fonte dos dados empregados. A discussão dos resultados faz parte da sexta seção, e as considerações finais são destacadas na última seção.

\section{Mercado do mel natural brasileiro}

Segundo informações do IBGE (2011), considerando-se a série de tempo entre 1974 e 2010, o Brasil possui o acumulado absoluto de mais de 678,7 mil toneladas de mel natural produzido, tornando a produção brasileira mais expressiva em meados do fim da última década do século XX e início da primeira década do século XXI.

No tocante às grandes regiões brasileiras, $\mathrm{o}$ Sul apresentou maior nível de produção absoluta de mel natural, com mais de 359,4 mil toneladas. Em seguida, a região Nordeste assumiu o segundo posto de maior produtor, com mais de 164,7 mil toneladas; o Sudeste, com mais de 128,2 mil, o Centro-Oeste, mais de 17,4 mil toneladas, e o Norte, com pouco mais de 9 mil, considerando o período supracitado (IBGE, 2011).

A partir desses dados, constata-se que a produção brasileira de mel natural possui um panorama capaz de classificá-la como concentrada, sobretudo nas regiões Sul, Nordeste e Sudeste, que respondem, respectivamente, por 52,93\%; $24,27 \%$ e $18,88 \%$ da produção apícola nacional, perfazendo, desta forma, o total de $96 \%$ da produção brasileira de mel natural. Ademais, é relevante destacar que o mel natural produzido no Brasil apresentou considerável variação em 2006, que pode ser atribuída ao fato de esse produto ter sofrido embargo europeu por falhas no controle de qualidade, que objetiva excluir a presença de resíduos no mel brasileiro e, com isso, garantir nível de qualidade elevado. Esta situação é apontada por Crespam e Scherer (2009, p. 13):

Em 17 de março de 2006, um dos principais compradores do mel nacional, a União Européia vetou a entrada do produto de procedência brasileira, sob a justificativa da ausência de controle e monitoramento de resíduos no produto brasileiro. Além disso, a União Européia utilizou-se da alegação de que o governo brasileiro não teria cumprido a exigência feita pelas autoridades sanitárias do bloco de realizar um controle rigoroso para detectar resíduos no produto. A medida de vetar a entrada do mel de procedência brasileira foi fundamentada em dois relatórios apresentados pelo Serviço Alimentar e Veterinário (FVO), que foram elaborados em 2003 e em 2005, a partir de visitas realizadas para inspecionar os processos de produção e monitoramento de produtos de origens animal e vegetal no país. 
Para reverter essa situação, o governo federal, por intermédio do Ministério de Agricultura, Pecuária e Abastecimento, desenvolveu controles de qualidades de resíduos adequados aos padrões de exigências da União Europeia. O monitoramento integrava também as ações do processo de controle de resíduos no mel brasileiro destinado à UE, com exames em amostras do mel produzido pelo Brasil (BUAINAIN e BATALHA, 2007).

Ao se comparar o Brasil com os dois principais fornecedores de mel para o mercado mundial (Argentina e China), observa-se que o Brasil apresenta uma posição confortável quanto à produção de mel natural, conforme Buainain e Batalha (2007, p. 129):

A apicultura brasileira conseguiu abrir as portas do mercado externo para o mel nacional graças, principalmente, aos problemas que assolaram os dois principais fornecedores de mel para o mercado mundial: a China e a Argentina. A China, maior produtora do mundo, teve suas exportações de mel suspensas pela União Européia após ser detectada a presença de clorofenicol em seu produto. Os apiários argentinos sofreram em decorrência da cria pútrida uma doença que ataca as abelhas e não tem cura.

Quando se realizam comparações entre o total produzido e exportado pelo Brasil e suas regiões, informações entram em conflito, conforme se verifica pela Tabela 1, a seguir. Por um lado, o rótulo de maior produtor pertence à região Sul; por outro, a região com maior volume de exportação é a Sudeste.

Diante deste fato, surgem as seguintes inquietações: i) até que ponto o mercado brasi- leiro (seja local, estadual, regional ou nacional) consumidor de mel natural influencia no total exportado deste produto? ii) do total produzido que excede o volume de mel exportado, quanto e qual o destino: consumidor final ou indústrias de beneficiamento?

A região Sul, ao longo do período analisado, reduziu percentualmente a participação no total de mel produzido pelo Brasil, passando de 58\% em 2000, para 43,49\% em 2010. Tal realidade tornou-se ainda mais crônica na evolução da representação do volume exportado pela região sulista, que caiu de 91,35\% em 2000 para $29,16 \%$ em 2010. A região Sudeste auferiu resultados de maior destaque na participação no total de mel comercializado internacionalmente: em 2000, representava apenas 5\% deste total e, em 2010, foi a região que mais exportou mel brasileiro, com 31\%. Com relação ao total produzido, apesar de ter elevado o volume, a região Sudeste reduziu sua participação de $20 \%$ em 2000 , para 16\% do total em 2010.

A região Nordeste, integrante do grupo que concentra, junto a Sul e Sudeste, a quantidade produzida e exportada de mel natural brasileira, além de ter aumentado o total produzido entre 2000 e 2010, dobrou sua participação no total produzido pelo Brasil, saindo de $17 \%$ para $34 \%$ no período analisado. O Centro-Oeste e Norte brasileiro, conjuntamente, em 2000, foram responsáveis por mais de $4 \%$ do total de mel natural produzido pelo Brasil, alcançando, em 2010, $6 \%$ de participação. Nesse mesmo período, suas exportações de mel foram insignificantes, não conseguindo, desta forma, promover assiduidade anual com a venda de mel natural para os mercados internacionais. 


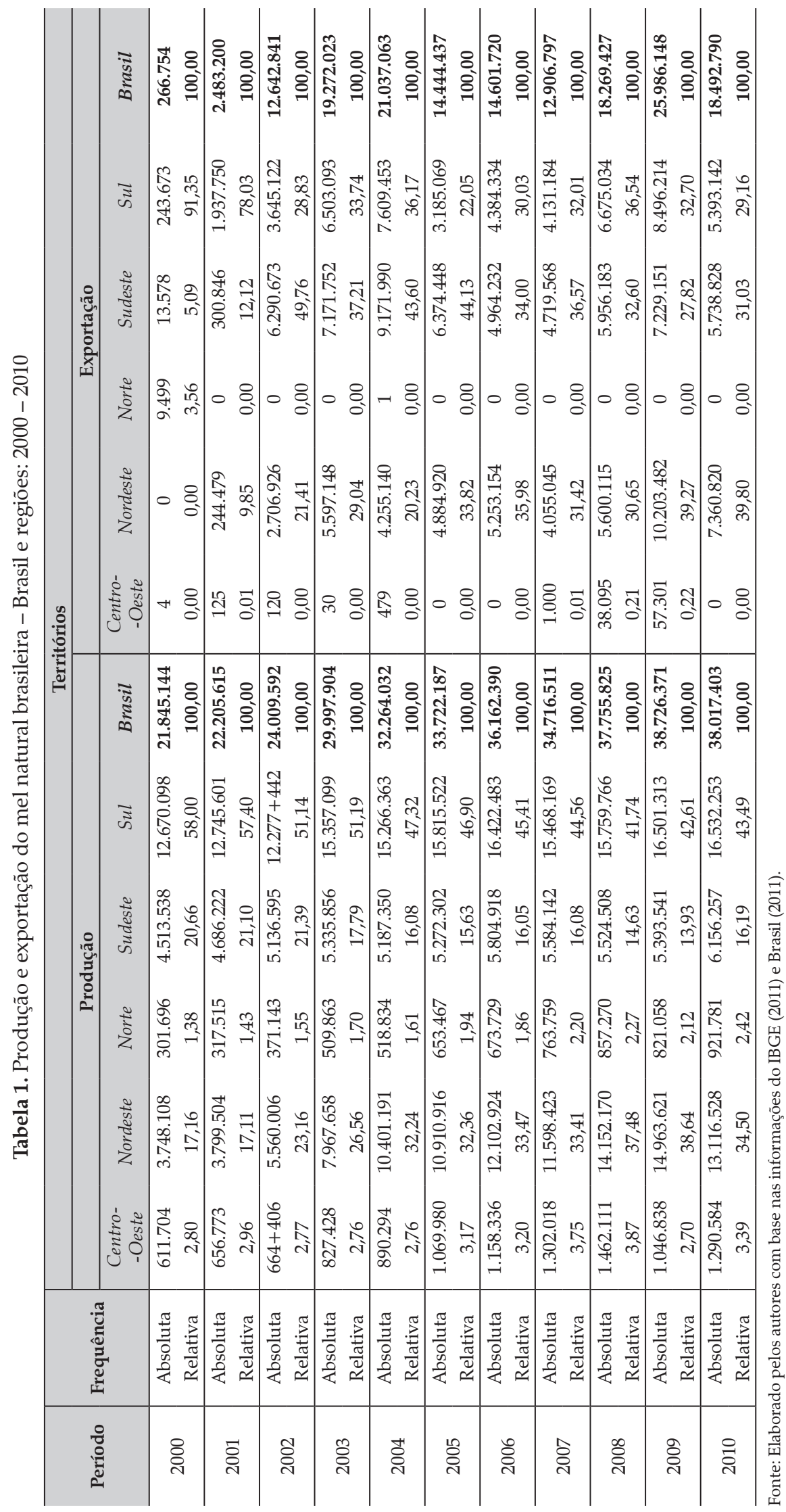




\section{Referencial teórico}

Um dos pressupostos da Lei do Preço Único baseia-se na perfeita simetria das trocas entre os mercados geograficamente separados, eliminando qualquer vestígio da presença de custos capazes de impactar negativamente as transações entre os mercados. A existência de custos de transação resulta em falha de mercado, pois implica que a informação com relação ao preço não seja perfeita. Como as decisões de compras e vendas feitas pelos agentes são baseadas no diferencial de preços entre mercados e no custo real com que se defronta, este diferencial de preços tem de ser positivo e suficiente para garantir o lucro do arbitrador (MALTSOGLOU e TANYERI-ABUR, 2005).

Barrett (2001) adota o termo custos de transação por meio de uma expressão algébrica, similar à expressão (1), decompondo o que considera ser uma boa caracterização de todos os custos envolvidos no processo:

$$
r_{\mathrm{ij}} \equiv f_{\mathrm{ij}}+v_{\mathrm{ij}}+d_{\mathrm{ij}}+w_{\mathrm{ij}}
$$

em que os custos de transação $\left(r_{\text {ij }}\right)$ são constituídos por custo de transporte $\left(f_{\mathrm{ij}}\right)$, custos variáveis $\left(v_{\mathrm{ij}}\right)$ relacionados às taxas, seguro de cargas, contratos, despesas financeiras, hedging, atendimento a barreiras técnicas (padrões sanitários e fitossanitários), entre outros, por impostos aduaneiros $\left(d_{\mathrm{ij}}\right)$ e também por custos não mensuráveis $\left(w_{\mathrm{ij}}\right)$, como o custo de oportunidade do empresário, custo de busca por informações, prêmios de risco associados às falhas nos contratos etc.

Para González-Rivera e Helfand (2001), os custos de transação devem considerar custos legais e de negociação e execução de contratos, custos financeiros, impostos, custos de transporte e de oportunidade relativos ao tempo destinado à busca de informações.

O modelo threshold cointegration, desenvolvido inicialmente por Balke e Fomby (1997), é uma maneira de combinar não linearidade $e$ cointegração. A estimativa dos parâmetros de threshold permite ao pesquisador calcular a magnitude da diferença entre o excedente e o nível de ajuste necessário para equilibrar, no longo prazo, os preços dos mercados espacialmente localizados. A não linearidade entre séries de preços de mercados espacialmente separados, identificadas pelo efeito threshold em determinado período de tempo, pode ser expressa conforme ilustrado na Figura 1.

Figura 1. Efeito threshold entre duas séries de preços

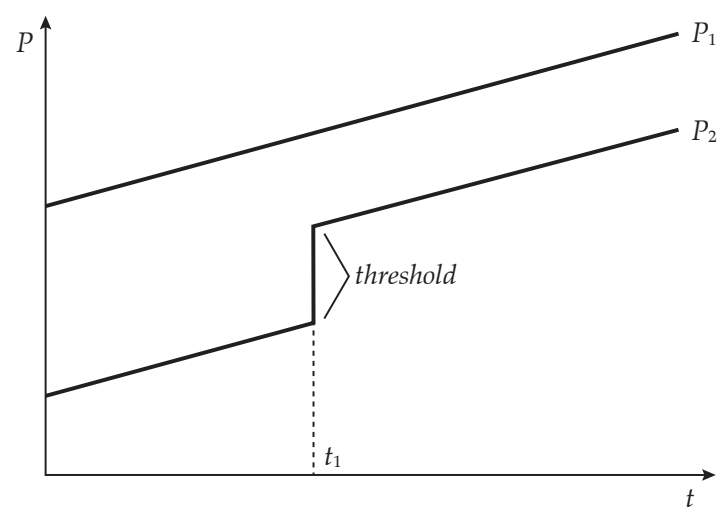

Fonte: Siqueira (2007, p. 34).

De acordo com Siqueira (2007), essa figura ilustra o efeito threshold entre duas séries de preços. Conforme se percebe, ambas as séries exibem uma tendência comum antes e após o período $t_{1}$. No entanto, no momento $t_{1}$, esta tendência se altera, isto é, há uma relação de cointegração antes de $t_{1}$ e outra relação de cointegração após este período. Assim, se um dado estudo estiver utilizando um modelo de cointegração simples, pode não detectar a presença da cointegração. Por outro lado, se o efeito threshold for incluído na análise, verifica-se a existência de cointegração. Desta forma, o modelo threshold aparece como uma alternativa para o modelo de cointegração, ou seja, se recomenda verificar a existência de efeito threshold quando não se encontra uma relação de cointegração entre as séries analisadas.

Em conformidade com Goodwin e Piggot (2001), os thresholds estimados são análogos aos custos de transação e consistentes com a expectativa de que quanto maior a distância entre os mercados, maiores são os thresholds.

Segundo Balcombe e Rapsomanikis (2008), o comportamento do threshold e ajustamentos assi- 
métricos não são sinônimos. A regulação de assimetrias pode, em geral, ser caracterizada como uma abordagem threshold. No entanto, existem formas assimétricas de ajustamento, não dependentes estritamente do conceito de um threshold. Os autores relatam ainda que a mudança de um regime para outro não pode ser repentina, mas gradual, uma vez que os agentes econômicos podem precisar de tempo para se adaptar às mudanças no ambiente econômico ou políticas.

Ademais, destaca-se a possibilidade de ocorrer arbitragem considerando-se os custos de transação, conforme sinalizado por Balcombe et al. (2007). Esses autores expõem essa condição, na qual incorporam, na análise, o tempo para ocorrer o custo de transação de um mercado para outro, considerando os seus preços. Desta forma:

a) as relações comerciais se desenvolvem de um mercado $A$ para um mercado $B$ qualquer, em um ambiente arbitrário, considerando a seguinte relação: $P_{t}^{A}+T_{t}^{A, B} \leq P_{t}^{B}$;

b) não haverá barganhas de lucros em meio à arbitragem entre os mercados, podendo ocasionar a ruptura do comércio ente $A$ e $B$, quando: $P_{t}^{A}+T_{t}^{A, B} \succ P_{t}^{B}$; e

c) os mercados referenciados poderão ser considerados integrados na presença da relação $P_{t}^{A}+T_{t}^{A, B} \succ P_{t}^{B}$ quando, no longo prazo, for constatado $P_{t}^{A}+T_{t}^{A, B} \leq P_{t}^{B}$, devido à ausência de comércio ou se houver falhas de comercialização decorrentes da abundância relativa de bens em cada mercado.

\section{Revisão de literatura}

Dentre os trabalhos que utilizaram modelos de integração espacial com threshold, podem-se citar Goodwin e Piggot (2001), Meyer (2004), Sarno et al. (2004), Balcombe et al. (2007) e Sanogo (2008) na literatura econômica internacional.

$\mathrm{Na}$ literatura econômica brasileira, essa abordagem foi empregada, por exemplo, por Siqueira (2007), que analisou o mercado de leite nos estados brasileiros de Goiás, Mato Grosso,
Mato Grosso do Sul, Minas Gerais, Paraná, Rio de Janeiro, Rio Grande do Sul, Santa Catarina e São Paulo com dados compreendendo o intervalo de tempo entre julho/1994 e setembro/2005. Os resultados desse estudo revelaram que o mercado brasileiro possui baixa velocidade de ajustamento a choques e não existe uma liderança na formação de preço do leite no Brasil.

Mattos (2008) avaliou a integração espacial dos mercados brasileiros de frango inteiro e resfriado, para o período de janeiro/1998 a junho/2007, considerando as praças de venda com maior potencial de comercialização (São Paulo - SP, Descalvado - SP, Porto Alegre - RS, Litoral Catarinense - SC, Oeste Paranaense - PR, Ponta Grossa - PR, Belo Horizonte - MG, Goiânia - GO, Fortaleza - CE, Recife - PE e Belém - PA). Os resultados demonstraram que: i) os parâmetros de threshold estimados indicaram que o processo de transmissão de preços é assimétrico; ii) há maior facilidade de transmitir aumentos de preços ocorridos em São Paulo aos demais mercados do que transmitir reduções de preços; iii) há elevados custos de transação, provavelmente decorrentes dos custos associados ao frete e à alta perecibilidade da carne de frango.

A integração espacial do mercado de boi gordo, considerando a presença de custos de transação, foi objeto de estudo de Mattos et al. (2009) nos estados de Minas Gerais e São Paulo, para o período de janeiro/1972 a agosto/2005. Os resultados verificados por esses autores denotaram a significância dos custos de transação entre os mercados estudados. A integração espacial do mercado do boi gordo brasileiro também foi analisada por Cunha et al. (2010) que, além de considerar esses dois estados supracitados, contemplou os estados do Espírito Santo, Rio de Janeiro, Paraná, Santa Catarina, Rio Grande do Sul, Mato Grosso, Mato Grosso do Sul e Goiás no período de 1994 a 2008. Os resultados desse estudo indicaram que tais mercados são integrados, e foi confirmada presença de assimetrias na transmissão de preços. Ademais, foram encontradas evidências de que choques negativos e/ou de baixa magnitude foram eliminados de maneira 
distinta dos positivos. Esses autores concluíram que os custos de transação influenciaram a ligação espacial entre os mercados.

Alves e Lima (2010) estudaram a integração espacial dos mercados brasileiros de açúcar, levando em conta os custos de transação, no período de maio de 2003 a dezembro de 2008. Os mercados analisados foram Alagoas, Pernambuco, São Paulo (Araçatuba e Ribeirão Preto), Paraná (Maringá) e Minas Gerais (Triângulo Mineiro). Os resultados apontaram a presença de significativos custos de transação nos pares de mercados de açúcar, sendo o maior threshold entre Alagoas e Ribeirão Preto e o menor, entre Araçatuba e Ribeirão Preto.

Por sua vez, Cunha e Sousa (2010) analisaram a integração espacial do mercado de melão nos estados nordestinos do Rio Grande do Norte, Ceará, Bahia, Pernambuco, Alagoas e Paraíba, no período de 2001 a 2009. Esses autores verificaram que houve equilíbrio de longo prazo entre os preços do melão nesses estados supracitados, excetuando-se a série de preços de Pernambuco. Ademais, constataram assimetria na transmissão de preços nos dois modelos estimados nos mercados analisados; portanto, o método empregado de cointegração com threshold foi apropriado para esse estudo. Entretanto, apesar da presença dessas assimetrias, as magnitudes dos valores dos threshold indicaram que os custos de transação não comprometeram sobremaneira a integração entre os mercados atacadistas de melão no Nordeste brasileiro.

De posse da revisão de literatura relacionada à análise de integração espacial entre mercados, considerando-se a presença de custos de transação, é possível dispor de um maior grau de entendimento sobre as relações entre mercados geograficamente separados, quando comparado com o relacionamento fundamentado na teoria da Lei do Preço Único. No entanto, não seria despretensioso frisar a prematuridade de afirmar que a complexidade de todo o dinamismo que permeia a integração dos mercados espacialmente separados poderia ser captado em sua totalidade por essa modelagem, capaz de valorar assimetrias representadas sobre a forma de custos de transação.

\section{Metodologia}

\subsection{Modelos analíticos}

Não incorporar os custos de transação nas análises de cointegração de mercados é não reconhecer a necessidade de estudos com modelagem dinâmica e dotada de maior poder de penetração das características inerentes e particulares das regiões consideradas. Desta forma, para atender os objetivos propostos neste estudo, empregou-se o modelo de cointegração com threshold, por meio dos modelos autorregressivos com threshold (TAR) e momentum-threshold (M-TAR), que foram operacionalizados pelos softwares Eviews 5.0 e RATS.

Antes de apresentar tais modelos analíticos, é relevante mencionar quando as séries são cointegradas e os testes que detectam a presença de cointegração. Segundo Engle e Granger (1987), caso duas séries não estacionárias formem um vetor de coeficientes que gerem resíduos estacionários, diz-se que estas séries são cointegradas. Dentre os testes para verificar a cointegração, conforme Margarido (2004), um dos mais utilizados devido à facilidade de sua aplicação é o teste denominado de Engle-Granger.

De acordo com Engle e Granger (1987), duas variáveis $Y_{1 \mathrm{t}}$ e $Y_{2 \mathrm{t}}$, consideradas integradas de ordem $d$, admitindo (d) como o total de diferenças necessárias para transformar as séries temporais em estacionárias, será cointegrado se for identificado um vetor $\delta$, caracterizado conforme a equação 2:

$$
\mu_{t}=\delta_{1} Y_{1 t}+\delta_{2} Y_{2 t}
$$

em que: $\mu_{t} \sim I(d-b)$, com $b \succ 0$, então $Y_{1 \mathrm{t}}$ e $Y_{2 \mathrm{t}}$ consideradas integradas de ordem $(d, b)$. Caso os resíduos $e_{\mathrm{t}}$ sejam estacionários $(d-b=0)$, haverá cointegração entre as variáveis integrantes do modelo, sinalizada por meio de uma relação de equilíbrio de longo prazo.

No cômputo da averiguação de existência ou não de raiz unitária, se houver a rejeição da hipótese nula, verifica-se cointegração entre duas séries temporais consideradas e a tendência 
de os mercados serem integrados. No entanto, observando a presença de custos de transação, é possível destacar que, caso o preço de um bem " $c$ " qualquer em um mercado " $a$ ", somado com o custo de transação relacionado com a transferência do bem do mercado " $a$ " para um mercado " $b$ ", seja maior que o preço deste bem " $c$ " neste segundo mercado, não haverá incentivo de ação de arbitradores. Com isso, ainda que os preços sejam cointegrados, não serão considerados mercados integrados (CUNHA et al., 2010).

Conforme sugerido por esses autores, o método mais apropriado para analisar a integração de mercados levando em conta a presença de não linearidades e descontinuidades no relacionamento entre os preços é a cointegração com a presença de threshold.

Considerando-se que os custos de transação não são inseridos explicitamente na proposta arquitetada por Engle e Granger (1987), eles fazem parte do termo de erro.

Assim, este estudo especificará $\mu_{\mathrm{t}}$ como um processo autorregressivo com threshold (TAR), seguindo a indicação de Enders e Granger (1998) e Enders e Siklos (2001). Desta forma, a série de resíduos pode ser reescrita como:

$$
\Delta \hat{\mathcal{U}}_{t}=\rho_{1} I_{t} \hat{\mathcal{u}}_{t-1}+\rho_{2}\left(1-I_{t}\right) \hat{\mathcal{U}}_{t-1}+\varepsilon_{t}
$$

em que: $\hat{u}_{\mathrm{t}}$ : série de resíduos, obtida através do teste Engle e Granger (1987); $I_{t}$ : variável dummy, que terá valor 1 , caso $\hat{u}_{t-1} \geq \tau$; e 0 se $\hat{u}_{t-1} \prec \tau$; $\tau$ : valor do threshold; $\varepsilon_{\mathrm{t}}$ : termo de erro independente de $\hat{u}_{\mathrm{t}}$ e $\tau$, em que se admite média zero, variância constante e ausência de autocorrelação serial.

Os valores de $\rho_{1}$ e $\rho_{2}$ assimilam o ajustamento assimétrico, compreendendo, desta forma, que, caso $\hat{u}_{\mathrm{t}-1}$ seja positivo, o ajuste acontecerá por meio de $\rho_{1} \hat{u}_{\mathrm{t}-1} ;$ no entanto, caso seja negativo, será captado por $\rho_{2} \hat{u}_{\mathrm{t}-1}$. Desta forma:

$$
\rho= \begin{cases}\rho^{(1)}, & \text { se }\left|\hat{u}_{t-1}\right| \leq \gamma \\ \rho^{(2)}, & \text { se }\left|\hat{u}_{t-1}\right| \succ \gamma\end{cases}
$$

em que: $\gamma$ representa assimetria (threshold) que delimita $\rho^{\mathrm{j}},(j=1,2, \ldots, n)$ indica que $\rho$ varia de acordo com o regime.
No modelo TAR, considera-se como hipótese nula a não existência de cointegração entre os mercados: $H_{0}: \rho_{1}=\rho_{2}=0$. Caso a hipótese nula de não cointegração seja rejeitada, testar-se-á a hipótese de ajustamento simétrico, empregando o teste $\mathrm{F}$ convencional.

Para averiguar com mais precisão se um choque positivo tenderá a persistir ou um choque negativo reverterá rapidamente ao threshold, será realizada refinação do modelo por meio do modelo momentum-threshold (M-TAR).

O modelo que assume a forma de um momentum-threshold (M-TAR) pode ser representado por:

$$
\Delta \hat{u}_{t}=\rho_{1} M_{t} \hat{u}_{t-1}+\rho_{2}\left(1-M_{t}\right) \hat{u}_{t-1}+\varepsilon_{t}
$$

em que: $M_{\mathrm{t}}$ é uma variável dummy que possuirá valor 1 , caso $\Delta \hat{\mathcal{u}}_{t-1} \geq \tau$, e será atribuído valor 0 , se $\Delta \hat{u}_{t-1} \prec \tau$.

Segundo Cunha et al. (2010, p. 258), ao citar Ewing et al. (2006), o modelo M-TAR pressupõe que $\hat{u}_{\mathrm{t}}$ exiba um momentum maior numa direção do que na outra; e o ajustamento será modelado por $\rho_{1} \hat{u}_{\mathrm{t}-1}$, caso $\Delta \hat{u}_{\mathrm{t}}$ esteja em nível superior ao threshold e por $\rho_{2} \hat{u}_{\mathrm{t}-1}$, caso $\Delta \hat{u}_{\mathrm{t}}$ esteja abaixo.

\subsection{Fontes dos dados}

Os dados utilizados neste trabalho contemplam as séries mensais de preços de exportação (US\$/t) do mel natural nos estados brasileiros do Rio Grande do Sul, Santa Catarina, Piauí, Ceará e São Paulo, obtidos junto ao Ministério do Desenvolvimento, Indústria e Comércio Exterior (BRASIL, 2011) no período entre janeiro de 2002 e julho de 2011. Vale destacar que foram considerados na análise os cinco estados brasileiros com maior volume exportado de mel natural no Brasil em 2010, perfazendo 87,60\%. Essas séries, respectivamente, são representadas por LPRS (Logaritmo natural do preço no estado do Rio Grande do Sul), LPSC (Logaritmo natural do preço no estado de Santa Catarina), LPPI, (Logaritmo natural do preço no estado do Piauí), LPCE (Logaritmo natural do preço no estado do Ceará) e LPSP (Logaritmo natural do preço no estado de São Paulo). 


\section{Resultado e discussão}

Para analisar a integração espacial dos mercados exportadores de mel natural no Brasil, antes de proceder aos testes de cointegração, deve-se verificar se essas séries de preços consideradas são estacionárias.

A estacionariedade foi detectada por meio da realização do teste de raiz unitária de DickeyFuller Aumentado (ADF), em que se constatou que todas as séries de preços avaliadas não foram estacionárias em nível; porém, passaram a ser estacionárias em primeira diferença, portanto, são integradas de ordem um, I(1). Entretanto, como o teste ADF apresenta baixo poder preditivo, empregaram-se também os testes de raiz unitária propostos por Phillips- Perron, indicados por PP, e Kwiatkowski, Phillips, Schmidt e Shin, denominado KPSS, e ambos os testes corroboraram o resultado encontrado pelo teste ADF, ou seja, apenas uma diferenciação foi suficiente para tornar as séries estacionárias, considerando as defasagens baseadas no critério de SIC (Schwarz Info Criterion).

Com o intuito de obter resultados dos testes de raiz unitária mais robustos, empregou-se o teste HEGY de raiz unitária sazonal, proposto por Hylleberg et al. (1990). Na especificação do teste, foi incluído um termo de intercepto, e as defasagens foram escolhidas por meio do critério de Schwartz. Os dados da Tabela 2 evidenciam o indicativo de ausência de raízes unitárias sazonais em todas as séries, conforme mostrada pela significância estatística de $\pi$. Além disso, não se identificou raiz unitária semianual nem raízes unitárias complexas nos pares examinados de $\pi_{\text {i's }}$. Desta forma, os preços do mel natural exportado pelo Brasil nos estados analisados são estacionários em primeira diferença, rejeitando a hipótese de raiz unitária sazonal.

Em seguida, buscou-se identificar o mercado que é formador de preços por meio do teste de exogeneidade fraca. Conforme se verifica pela Tabela 3, o mercado exportador de mel natural do Rio Grande do Sul caracteriza-se como exógeno fraco, uma vez que não se rejeita a hipótese de que esse mercado não seja influenciado no longo prazo pelos demais mercados analisados. Portanto, constata-se que o estado gaúcho atua como mercado central, capaz de influenciar mudanças nos preços do mel natural dos estados de Santa Catarina, Piauí, Ceará e São Paulo no longo prazo, já que se rejeitou a hipótese nula de mercado exógeno fraco nesses estados, com probabilidade a $1 \% ; 1 \% ; 5 \%$ e $10 \%$, respectivamente.

Tabela 2. Teste de identificação da presença de raízes sazonais nas séries de preços mensais do mel natural exportado pelos estados considerados na análise, de janeiro de 2002 a julho de 2011

\begin{tabular}{cccccc}
\hline \multirow{2}{*}{ Frequência/Coeficientes } & \multicolumn{4}{c}{ Séries } \\
\cline { 2 - 6 } & LPRS & LPSC & LPPI & LPCE & LPSP \\
\hline Lags $^{(\mathrm{a})}$ & 0 & 0 & 0 & 0 & 0 \\
$\pi_{1}=0$ & $1,87^{(\mathrm{b})}$ & $2,80^{(\mathrm{b})}$ & $2,39^{(\mathrm{b})}$ & $2,61^{(\mathrm{b})}$ & $2,35^{(\mathrm{b})}$ \\
$\pi_{2}=0$ & $-2,48^{* *}$ & $-2,41^{* *}$ & 1,44 & $-2,64^{* * *}$ & $-1,64^{*}$ \\
$\pi_{3}=\pi_{4}=0$ & $6,00^{* * *}$ & $6,59^{* * *}$ & 1,40 & $4,22^{* *}$ & 0,55 \\
$\pi_{5}=\pi_{6}=0$ & $14,42^{* * *}$ & $17,84^{* * *}$ & $17,41^{* * *}$ & $12,58^{* * *}$ & $10,68^{* *}$ \\
$\pi_{7}=\pi_{8}=0$ & $10,10^{* * *}$ & $11,06^{* * *}$ & $10,78^{* * *}$ & $12,92^{* * *}$ & $7,94^{* * *}$ \\
$\pi_{9}=\pi_{10}=0$ & $9,80^{* * *}$ & $12,47^{* * *}$ & $14,30^{* * *}$ & $13,87^{* * *}$ & $10,42^{* * *}$ \\
$\pi_{11}=\pi_{12}=0$ & $9,68^{* * *}$ & $13,99^{* * *}$ & $10,57^{* * *}$ & $8,79^{* * *}$ & $10,13^{* * *}$ \\
$\pi_{1}=\ldots=\pi_{12}=0$ & $62,65^{* * *}$ & $57,09^{* * *}$ & $30,42^{* * *}$ & $36,94^{* * *}$ & $8,13^{* * *}$ \\
$\pi_{2}=\ldots=\pi_{12}=0$ & $68,34^{* * *}$ & $62,26^{* * *}$ & $33,18^{* * *}$ & $40,29^{* * *}$ & $8,38^{* * *}$ \\
\hline
\end{tabular}

Nota: (a) O número de defasagens foi baseado no critério de Schwarz; (b) Indica não rejeição da hipótese nula; $\left.\left({ }^{* * *}\right),{ }^{* *}\right)$ e $\left(^{*}\right)$ indicam rejeição de $H_{0}$ a 1\%,5\% e 10\%, respectivamente, cujos valores críticos foram obtidos por Franses e Hobijn (1997).

Fonte: Elaborado pelos autores com base nos resultados da pesquisa. 
Tabela 3. Teste de exogeneidade fraca realizado para as variáveis LPRS, LPSC, LPPI, LPCE e LPSP, de janeiro de 2002 a julho de 2011

\begin{tabular}{lcc}
\hline \multicolumn{1}{c}{ Mercados potencialmente exógenos } & Estatística de teste & P-valor \\
\hline Rio Grande do Sul (LPRS) & $0,0831^{\mathrm{NR}}$ & 0,7732 \\
Santa Catarina (LPSC) & $11,8337^{* * *}$ & 0,0006 \\
Piauí (LPPI) & $22,4341^{* * *}$ & 0,0000 \\
Ceará (LPCE) & $4,8961^{* *}$ & 0,0269 \\
São Paulo (LPSP) & $3,5455^{*}$ & 0,0597 \\
\hline
\end{tabular}

Notas: ${ }^{\mathrm{NR}}$ Indica não rejeição da hipótese nula a $\left.10 \% ;{ }^{* * *}\right),\left({ }^{* *}\right) \mathrm{e}\left({ }^{*}\right)$ indicam rejeição de $H_{0}$ a $1 \%, 5 \%$ e $10 \%$, respectivamente. Fonte: Elaborado pelos autores com base nos resultados da pesquisa.

$\mathrm{O}$ resultado desse teste que aponta que o estado do Rio Grande do Sul, como mercado central na formação dos preços de exportação do mel natural, está consistente com os argumentos de que esse estado tem representado a maior variação percentual de crescimento do total de mel natural exportado no período enfocado e, historicamente, por esse estado ter se destacado como líder brasileiro da produção de mel.

Identificado o mercado central, foram estimadas, pelo método dos Mínimos Quadrados Ordinários (MQO), as equações de cointegração entre os preços de exportação do mel natural nos estados analisados. Essas relações de longo prazo foram realizadas entre o preço estabelecido no mercado central, representado pelo Rio Grande do Sul, e os demais estados considerados (Santa Catarina, Piauí, Ceará e São Paulo), segundo as orientações da metodologia de Engle e Granger (1987), cujos resultados se encontram na Tabela 4.
Os coeficientes relativos à elasticidade de transmissão de preço de longo prazo foram estatisticamente significativos a $1 \%$ de significância. No tocante às elasticidades, percebeu-se que uma variação de $10 \%$ no preço de exportação do mel natural no Rio Grande do Sul causaria uma mudança, no mesmo sentido, de $10,07 \%$ no preço de Santa Catarina, de 8,95\% no Piauí, 9,87\% no Ceará e 10,07\% em São Paulo.

Conforme identificado por Mattos (2008) e Cunha et al. (2010), verificou-se também que existem indicativos de uma relação inversa entre o grau de transmissão de preços e a distância física entre os mercados, pois, por um lado, os estados brasileiros exportadores de mel natural com maior volume comercializado e localizado, segundo um menor distanciamento físico com o território gaúcho, Santa Catarina e São Paulo, apresentaram maiores coeficientes de elasticidades, 1,0074 e 1,0076, respectivamente. Por outro

Tabela 4. Relações de cointegração entre o preço do Rio Grande do Sul e os mercados de Santa Catarina (SC), Piauí (PI), Ceará (CE) e São Paulo (SP), de janeiro de 2002 a julho de 2011

\begin{tabular}{lcc}
\hline \multicolumn{1}{c}{ Mercados } & Equação de cointegração & $P_{\mathrm{i}, \mathrm{t}}=\beta_{0}+\beta_{0} P_{\mathrm{RSt}}+\mu_{\mathrm{t}}$ \\
\hline \multirow{2}{*}{ Rio Grande do Sul e Santa Catarina } & $S C_{\mathrm{i}}=0,0131+1,0074 R S_{\mathrm{t}}$ & $R_{\text {ajustado }}^{2}=0,93$ \\
& $(0,0181)(0,0241)$ & $D W=1,61$ \\
\hline \multirow{2}{*}{ Rio Grande do Sul e Piauí } & $P i_{\mathrm{i}}=0,0633+0,8953 R S_{\mathrm{t}}$ & $R_{\text {ajustado }}^{2}=0,89$ \\
& $(0,0215)(0,0286)$ & $D W=1,89$ \\
\hline \multirow{2}{*}{ Rio Grande do Sul e Ceará } & $C E_{\mathrm{i}}=0,0723+0,9870 R S_{\mathrm{t}}$ & $R_{\text {ajustado }}^{2}=0,86$ \\
& $(0,0270)(0,0360)$ & $D W=1,45$ \\
\hline \multirow{2}{*}{ Rio Grande do Sul e São Paulo } & $S P_{\mathrm{i}}=0,0154+1,0076 R S_{\mathrm{t}}$ & $R_{\text {ajustado }}^{2}=0,82$ \\
& $(0,0328)(0,0437)$ & $D W=1,78$ \\
\hline
\end{tabular}

Nota: Os valores entre parênteses referem-se ao erro padrão do parâmetro estimado; DW: refere-se à estatística de Durbin-Watson após a correção de correlação serial dos resíduos.

Fonte: Resultados da pesquisa. 
lado, estados mais longínquos e com representativo volume de mel natural exportado no período considerado em análise, como Ceará $(0,98)$ e Piauí $(0,89)$, apresentaram coeficientes de elasticidade menores.

Os resultados das Tabelas 5 e 6 permitem verificar se, de fato, os custos de transação causam descontinuidades no processo de transmissão de preços. A primeira hipótese averiguada diz respeito à investigação da ausência de cointegração entre as séries de preços dos mercados analisados, testando se $\rho_{1}=\rho_{2}=0$. As informações estruturadas na Tabela 4 caracterizam o mercado brasileiro formado pelos cinco estados detentores de maior volume exportado de mel como integrado, pois há a existência de um equilíbrio de longo prazo entre as séries de preços estudadas. As modelagens TAR e M-TAR legitimaram tal configuração em decorrência de todas as relações indicadas, por meio do fato de os pares entre os estados analisados rejeitarem a referida hipótese, considerando $1 \%$ de significância.

Ainda segundo informações presentes na Tabela 5, rejeitou-se a hipótese nula $\rho_{1}=\rho_{2}$, que buscou verificar o ajustamento simétrico entre os integrantes do mercado analisado. O modelo tipo TAR e M-TAR rejeitou tal hipótese para todos os pares de mercado investigados, com nível de significância de $1 \%$. Com isso, cabe salientar o posicionamento assertivo desta pesquisa, que optou pela especificação não linear, reiterando a impor- tância das modelagens que consideram assimetrias na transmissão de preços, ou seja, utilizam o efeito threshold, em oposição à análise tradicional de Engle e Granger (1987).

A Tabela 6 contém a estimativa do parâmetro threshold e dos coeficientes dos modelos TAR e M-TAR. Os resultados do modelo TAR apontaram que choques inferiores a $0,58 \%$ no preço médio de Santa Catarina são eliminados com uma velocidade diferenciada da apresentada por choques maiores que esse percentual. A configuração apresentada pela região catarinense é distinta da exibida pelos mercados cearense e piauiense, dotados de maior distanciamento geográfico, no qual apresentaram, respectivamente, valor absoluto mais elevado, $2,06 \%$ e $1,77 \%$. Desta forma, essas relações reiteram a base teórica que relaciona o valor de um threshold mais elevado para estados dotados de maior distanciamento físico do mercado central. Essas respectivas velocidades são captadas pelos valores de $\rho_{1}$ e $\rho_{2}$.

Verificada com realidade bastante peculiar, a região paulista necessita de uma atenção particular. Tal necessidade é oriunda de uma configuração capaz de ratificar e contestar simultaneamente a proposição de Goodwin e Piggot (2001) no tocante à relação do distanciamento físico e valor do threshold. Por um lado, quando comparado com os resultados de Santa Catarina, o estado paulista alcança maior threshold em decorrência de possuir maior distanciamento físico

Tabela 5. Parâmetros estimados para os modelos TAR e M-TAR: teste das hipóteses, ausência de cointegração e ajustamento simétrico

\begin{tabular}{lcccc}
\hline \multirow{2}{*}{ Mercados } & \multicolumn{2}{c}{ Modelo TAR } & \multicolumn{2}{c}{ Modelo M-TAR } \\
\cline { 2 - 5 } & $\rho_{1}=\rho_{2}=0$ & $\rho_{1}=\rho_{2}$ & $\rho_{1}=\rho_{2}=0$ & $\rho_{1}=\rho_{2}$ \\
\hline \multirow{2}{*}{ Rio Grande do Sul e Santa Catarina } & $71,6621^{* * *}$ & $141,0618^{* * *}$ & $40,4490^{* * *}$ & $77,4518^{* * *}$ \\
& {$[9,02]$} & $\{0,0000\}$ & {$[9,02]$} & $\{0,0000\}$ \\
\hline \multirow{2}{*}{ Rio Grande do Sul e Piauí } & $29,9795^{* * *}$ & $51,4627^{* * *}$ & $10,1767^{* * *}$ & $15,3976^{* * *}$ \\
& {$[9,02]$} & $\{0,0000\}$ & {$[9,02]$} & $\{0,0000\}$ \\
\hline \multirow{2}{*}{ Rio Grande do Sul e Ceará } & $298036^{* * *}$ & $32,5064^{* * *}$ & $32,3175^{* * *}$ & $36,1244^{* * *}$ \\
& {$[9,02]$} & $\{0,0000\}$ & {$[9,02]$} & $\{0,0000\}$ \\
\hline \multirow{2}{*}{ Rio Grande do Sul e São Paulo } & $54,5464^{* * *}$ & $59,9919^{* * *}$ & $22,1209^{* * *}$ & $36,1692^{* * *}$ \\
& {$[9,02]$} & $\{0,0000\}$ & {$[9,02]$} & $\{0,0000\}$ \\
\hline
\end{tabular}

Notas: ${ }^{* * *}$ indica significativo a $1 \%$. Os valores entre colchetes correspondem aos valores tabelados da estatística $\Phi$ e $\Phi^{\mathrm{m}}(\mathrm{TAR}$ e M-TAR, respectivamente) e os entre chaves dizem respeito aos p-valores obtidos para o teste $\mathrm{F}$.

Fonte: Resultados da pesquisa. 
Tabela 6. Parâmetros estimados para os modelos TAR e M-TAR

\begin{tabular}{lcccccc}
\hline \multicolumn{1}{c}{ Mercados } & \multicolumn{3}{c}{ Modelo TAR } & \multicolumn{3}{c}{ Modelo M-TAR } \\
\cline { 2 - 6 } & $\hat{\tau}$ & $\rho_{1}$ & $\rho_{2}$ & $\hat{\tau}$ & $\rho_{1}$ & $\rho_{2}$ \\
\hline Rio Grande do Sul e Santa Catarina & 0,0058 & $\begin{array}{c}0,1117^{* * *} \\
(0,0111)\end{array}$ & $\begin{array}{c}-0,0623^{* * *} \\
(0,0095)\end{array}$ & 0,0567 & $\begin{array}{c}0,1238^{* * *} \\
(0,0156)\end{array}$ & $\begin{array}{c}-0,0555^{* * *} \\
(0,0131)\end{array}$ \\
\hline Rio Grande do Sul e Piauí & $-0,0206$ & $\begin{array}{c}0,1169^{* * *} \\
(0,0166)\end{array}$ & $\begin{array}{c}-0,0615^{* *} \\
(0,0181)\end{array}$ & $-0,0749$ & $\begin{array}{c}0,0813^{* * *} \\
(0,0198)\end{array}$ & $\begin{array}{c}-0,0569^{* *} \\
(0,0286)\end{array}$ \\
\hline Rio Grande do Sul e Ceará & $-0,0177$ & $\begin{array}{c}0,0689^{* * *} \\
(0,0092)\end{array}$ & $\begin{array}{c}0,0303^{* *} \\
(0,0145)\end{array}$ & 0,0073 & $\begin{array}{r}0,0698^{* * *} \\
(0,0089)\end{array}$ & $\begin{array}{c}-0,0314^{* *} \\
(0,0140)\end{array}$ \\
\hline Rio Grande do Sul e São Paulo & 0,0280 & $\begin{array}{c}-4,1985^{* * *} \\
(0,6555)\end{array}$ & $\begin{array}{c}-0,6209^{* * *} \\
(0,1021)\end{array}$ & 0,0639 & $\begin{array}{c}2,8486^{* * *} \\
(-0,6327)\end{array}$ & $\begin{array}{c}-0,4425^{*} \\
(0,2413)\end{array}$ \\
\hline
\end{tabular}

Notas: ***,** $\mathrm{e}$ * indicam, respectivamente, significativos a 1\%, 5\% e 10\%. Os valores entre parênteses referem-se ao erro padrão.

Fonte: Resultados da pesquisa.

com a região gaúcha (SC: 0,58\% e SP: $2,80 \%$ ). Por outro lado, considerando-se os estados do Ceará e Piauí, apesar de serem mais distantes do que o Rio Grande do Sul, em termos de localização, o threshold de São Paulo continuou em patamares mais elevados (CE: 1,77\%; PI: 2,06\%; SP: 2,80\%).

Com exceção do mercado cearense, observou-se que os demais apresentaram estimativas do parâmetro threshold em patamares mais elevados no modelo M-TAR do que as encontradas na modelagem TAR. Credenciada segundo a possibilidade de identificar discrepâncias no ajustamento a choques positivos e negativos no mercado central, a modelagem M-TAR, para todas as relações de pares de mercado, conforme Tabela 5, sinalizou que choques positivos, acima do threshold, foram eliminados mais rapidamente do que choques negativos.

Em Santa Catarina, elevações dos preços em patamar superior a 5,67\% do preço médio do estado foram removidas mais rapidamente do que quedas nos preços, em decorrência de $\left|\rho_{1}\right|>\left|\rho_{2}\right|$. No território piauiense, as elevações dos preços médios do mel natural capazes de extrapolar 7,49\% do preço médio do estado foram eliminadas mais rapidamente do que reduções nos preços, uma vez que $\left|\rho_{1}\right|>\left|\rho_{2}\right|$. Nos estados cearense e paulista, as elevações dos preços, segundo níveis mais elevados que $0,73 \%$ e $6,39 \%$, respectivamente, nos preços médios dos referidos estados, foram eliminadas mais rapidamente do que quedas nos preços, motivadas pela relação em que $\left|\rho_{1}\right|>\left|\rho_{2}\right|$.
Cabe salientar, ainda, que, com exceção do estado cearense, Santa Catarina e Piauí ratificaram a exposição teórica que relaciona distanciamento geográfico e valor do threshold, uma vez que o território piauiense, mais distante do Rio Grande do Sul, alcançou valor mais elevado, e a região catarinense, geograficamente mais próxima do mercado gaúcho, com menor threshold. São Paulo, conforme realidade exposta na modelagem TAR, ratificou ambiguidade no modelo M-TAR. Acredita-se que, em decorrência de o estado paulista comprar quantidades de mel em vários estados brasileiros, uma vez que as informações sobre o volume produzido ser inferior ao exportado, os acréscimos de custos envolvidos na transferência deste produto até São Paulo podem ser explicativos da presença de custos de transação em patamares mais elevados no mercado sudestino.

\section{Conclusões}

As abordagens convencionais de integração de mercados não consideram a existência dos custos de transação; porém, é importante que tais custos sejam incorporados à análise. Assim, para captá-los, este estudo adotou as abordagens TAR e M-TAR.

Com base nos resultados obtidos, constata-se que houve um relacionamento de equilíbrio de longo prazo entre o preço estabelecido no mer- 
cado central, representado pelo Rio Grande do Sul, e os demais estados considerados, tendo em vista que todos os parâmetros estimados foram significativos.

Portanto, conclui-se que o ajustamento de preços a eventuais choques ocorreu de forma diferente, dependendo da magnitude do desvio em relação ao equilíbrio de longo prazo. Ademais, verifica-se também assimetria na transmissão de preços, indicada nos modelos estimados para esses mercados. Desta forma, os custos de transação influenciam a interação espacial entre tais mercados, sinalizando que a utilização dessas ferramentas analíticas é apropriada para este estudo. No entanto, mesmo com a confirmação dessas assimetrias, nota-se que elas não comprometem fortemente a integração desses mercados estudados, uma vez que, em geral, as magnitudes dos valores dos threshold foram relativamente baixas.

\section{Referências}

ALVES, F. S. e LIMA, R. C. Integração dos mercados de açúcar no Brasil considerando a presença de custos de transação. In: Congresso Brasileiro de Economia e Sociologia Rural, 48, 2010. Anais... Campo Grande, MS: SOBER, 2010.

BALCOMBE, K., BAILEY, A. e BROOKS, J. Threshold effects in price transmission: the case of Brazilian wheat, maize and soya prices. American Journal of Agricultural Economics, v. 89, n. 2, p. 308-323, 2007.

BALCOMBE, K. G. e RAPSOMANIKIS, G. Bayesian estimation of non-linear vector error correction models: the case of the sugar-ethanol-oil nexus in Brazil. American Journal of Agricultural Economics. v. 90, n. 3, p. 658-668, 2008.

BALKE, N.S. e FOMBY, T.B. Threshold cointegration. International Economic Review, v. 38, p. 627-645, 1997.

BARRETT, C.B. Measuring integration and efficiency in international agricultural markets. Review of Agricultural Economics, v. 23, n. 1, p. 19-32, 2001.

BRASIL. Ministério de Desenvolvimento Indústria e Comércio - MDIC - ALICEweb. Valores e quantidades anuais de mel natural exportados no mercado: janeiro/1986 a dezembro/2010. Brasília-DF. Disponível em: < aliceweb. desenvolvimento.gov.br>. Acesso em: 12 fev. 2011.
BUAINAIN, A. M. e BATALHA, M. O. (Coord.). Cadeia produtiva de flores e mel. Brasília: IICA: MAPA/SPA, 2007.

CRESPAM, C. C. e SCHERER, F. L. Nem tudo são flores na produção e na exportação de mel: barreiras técnicas em foco. 2009. Disponível em: < http://www.ifbae.com.br/ congresso5/pdf/B0063.pdf>. Acesso em: 13 dez. 2011.

CUNHA, D. A. e SOUSA, E. P. Integração espacial dos mercados atacadistas de melão no nordeste considerando a presença de custos de transação. ). In: Congresso Brasileiro de Economia e Sociologia Rural, 48, 2010. Anais... Campo Grande, MS: SOBER, 2010.

., LIMA, J. E. e BRAGA, M. J. Integração espacial do mercado de boi gordo: uma análise de cointegração com threshold. Análise Econômica, Porto Alegre, v. 28, n. 53 , p. $251-267,2010$.

ENDERS, W. e GRANGER, C.W.J. Unit-root tests and asymmetric adjustment with an example using the term structure of interest rates. Journal of Business and Economic Statistics, n. 16, p. 304-311, 1998.

ENDERS, W. e SIKLOS, P. L. Cointegration and threshold adjustment. Journal of Business and Economic Statistics, n. 19, p. 166-176, 2001.

ENGLE, R. F. e GRANGER, C. W. J. Co-integration and error correction: Representation, estimation and testing. Econometrica, v. 55, n. 2, p. 251-276, mar. 1987.

EWING, B. T., HAMMOUDEH, S. M. e THOMPSON, M. A. Examining asymmetric behavior in US Petroleum futures and spot prices. The Energy Journal, v. 27, n. 3, p. 9-23, 2006.

FOOD Agriculture Organization United NationsFAO. FAOstat. Disponível em: <http://faostat.fao.org/ default.aspx>. Acesso em 18 fev. 2011.

FRANSES, P. H. e HOBIJN, B. Critical values for unit root tests in seasonal time series. Journal of Applied Statistics, v. 24, p. 25-46, 1997.

GONZÁLEZ-RIVERA, G. e HELFAND, S. M. Economic development and the determinants of spatial integration in agricultural markets. Riverside: Department of Economics, University of California, WorKing Paper, v. 1, n. 28, p. 1-31, 2001.

GOODWIN, B. K. e PIGGOTT, N. E. Spatial market integration in the presence of threshold effects. American Journal of Agricultural Economics, v. 83, n. 2, p. 302-317, 2001.

HYLLEBERG. S., ENGLE. R. F., GRANGER. C. W. J. e YOO. B. S. Seasonal integration and cointegration. Journal of Econometrics, v. 44, n. 1-2, p. 215-238, 1990. 
IBGE - Instituto Brasileiro de Geografia e Estatística. Sistema do IBGE de Recuperação Automática-SIDRA. Disponível em: <http://www.sidra.ibge.gov.br/bda/ pecua/> . Acesso em: out. 2011.

KHAN, A. S., VIDAL, M. F., LIMA, P. V. P. S. e BRAINER, M. S. C. P. Perfil da apicultura no Nordeste brasileiro. Fortaleza, CE: Banco do Nordeste do Brasil, 2014.

KRUGMAN, P. R. e OBSTFELD, M. International economics: theory and policy. 5. ed. Massachusetts: Addson Welsley, 2005.

MALTSOGLOU, I. e TANYERI-ABUR, A. Transactions costs, Institutions and smallholder market integration: potato producers in Peru. ESA Working Paper, n. 05/04, p. 1-13, 2005.

MARGARIDO, M. A. Teste de cointegração de Johansen utilizando o SAS®. Agricultura, SP, v. 51, n. 1, p. 87-101, 2004.

MATOS, V. D. A apicultura no Estado do Ceará: competitividade, nível tecnológico e seus fatores condicionantes, produção e exportação de mel natural. 192 p. Dissertação (Mestrado em Economia Rural) Universidade Federal do Ceará, Fortaleza, CE, 2005.

MATTOS, L. B. Efeitos de custos de transação sobre a integração espacial de mercados regionais de carne de frango no Brasil. 179 p. Tese (Economia Rural) - Universidade Federal de Viçosa, Viçosa, MG, 2008.

., LIMA, J. E. e LIRIO, V. S. Integração espacial de mercados na presença de custos de transação: um estudo para o mercado de boi gordo em Minas Gerais e São Paulo. Revista de Economia e Sociologia Rural. Piracicaba, v. 47, n. 01, p. 249-274, 2009.

MEYER, J. Measuring market integration in the presence of transaction costs - a threshold vector error correction approach. Agricultural Economics, v. 31, p. 327-334, 2004.

SANOGO, I. Spatial integration of the rice market: empirical evidence from mid-west and far-west Nepal and the Nepalese-Indian border. Asian Journal of Agriculture and Development, v. 4, n. 1, p. 139-156, 2008.

SARNO, L., TAYLOR, M. P. e CHOWDHURY, I. Nonlinear dynamics in deviations from the law of one price: a broad-based empirical study. Journal of International Money and Finance, v. 23, p. 1-25, 2004.

SIQUEIRA, K. B. The dynamics of farm milk price formation in Brazil. 143 p. Tese (Doutorado em Economia Rural) Universidade Federal de Viçosa. Viçosa, MG, 2007. 